\title{
Carer-centred care: general practice support to family caregivers
}

Michelle Pyer, PhD FRGS, Senior Researcher (corresponding author)

The University of Northampton

Boughton Green Road

Northampton

Northamptonshire

NN2 7AL

01604892831

michelle.pyer@northampton.ac.uk

Jackie Campbell, PhD MInstP CPhys CStat FCOT, Professor of Neurophysiology

Alison Ward, MSc, Researcher

The University of Northampton

Boughton Green Road

Northampton

Northamptonshire

NN2 7AL

Running title

Carer-centred care in general practice

Key words

Informal carers, familial carers, carer support, expert partners, general practice 


\begin{abstract}
Rationale, aims and objectives

In February 2009 the UK's Department of Health invited bids for funding to host demonstrator sites in order to test new approaches to working with and for carers within the national Carers'Strategy $[1 ; 2]$. This paper discusses some of the findings of the local level evaluation of one of the host sites, situated in Northamptonshire.
\end{abstract}

\title{
Methods
}

A mixed-method approach was used to gather data froma range of participant groups. This paper presents the findings of two of those methods; a postal survey of carers and an audit of support offered by general practices.

\section{Results}

The findings of the evaluation show a considerable improvement in the extent to which carers' roles were identified by their surgeries, however there is a need to ensure that this information is recorded and used in ways which benefit carers. Carers noted challenges in dealing with practice staff, particularly administrative staff who were not aware of their needs. Practices noted a range of services which were offered to carers, however carers were not always aware that these were available, suggesting that further publication of these opportunities is needed.

\section{Conclusions}

The paper argues the importance of the role undertaken by carers, and signals the range of ways in which General Practice can support or inhibit these activities.

\section{Introduction}

\section{National context}

The role(s) that carers ${ }^{1}$ play make a significant contribution to local communities and to society as a whole in the UK. The number of full time unpaid carers in the UK exceeds the size of the workforce in the NHS and continues to grow [3], showing the need for targeted efforts to support their endeavours. In order to implement effective, personalised approaches for both the cared for and those who undertake caring, there is a need to recognise not only the needs of carers as carers, but also as individuals and patients in their own right. This paper draws from the findings of an evaluation of the Carer Strategy Demonstrator Site (CSDS) in Northamptonshire (a national evaluation

\footnotetext{
${ }^{1}$ The term 'carer' represents a varied and complex set of needs and roles; there is no single carer 'profile' [1]. Here, the term 'carer' relates to those individuals who care on an informal basis, rather than those who are formally employed to do so.
} 
report of this programme is available [2]). The overarching aim of the study was to evaluate the implementation and effectiveness of the pilot Carer Strategy Demonst rator Site in Northamptonshire, with a view to informing its future development.

A number of factors can affect the likelihood of an individual becoming a carer including age, gender, ethnicity and employment situation $[4 ; 5)$. A majority of carers are related in some way to the person that they care for, whether this be a member of their immediate family or not [6]. The role(s) that carers undertake further differentiates their experiences, and in turn, their needs. Carers may provide a high level of practical, medical and emotional support for the person that they care for, support the person that they care for in accessing services, or may even take the lead in commissioning a personal package for them, becoming 'expert partners' $[7 ; 8]$. The profile of carers as experts in health has been raised within a number of policy documents. The accompanying handbook to the NHS Constitution [9] discusses the importance of treating carers as experts and partners. Similarly, the National Carers' Strategy promotes the significance of carers as expert care partners [1], signalling the developing profile of carers and the importance of their involvement in decision-making relating to the needs of the individual that they care for.

Whilst there are undoubtedly positive aspects of undertaking a caring role, including a sense of satisfaction and enjoyment [10] a wealth of literature also reports negative relationships between the health of a carer, the type of care that they provide, and the number of hours that they spend caring [11]. The support that carers receive from services is an important element in supporting health and wellbeing [3]. A lack of professional understanding of carer needs, limited recording of carer responsibilities and minimal information for carers accessing support are all barriers to carers accessing services in their own right [12]. The health of carers is important not just for carers in their own right; poor health can have an impact on their ability to care.

\section{Carer demonstrator sites}

In February 2009 the Department of Health invited bids for funding to host demonstrator sites aimed at testing new approaches to working with and for carers within the national Carers' Strategy [1]. Twenty-five sites were commissioned and a national evaluation undertaken [2]. Each site was also required to conduct a local evaluation of process and impact. The Northamptonshire demonstrator site was one of 25 across England, and one of seven Better NHS Support for Carers sites (others focused their efforts on the delivery of health checks or breaks for carers) [1]. The Northamptonshire project employed 
dedicated Carer Assessment and Support Workers (CASWs), trained to support carers and primary care professionals working with them. Their role was two-fold: to identify and support carers within the county, and to liaise with General Practice surgeries, to improve services for carers. This paper reports on changes during the timeframe that the CSDS site was in operation (evaluation period: August- November 2011) via a discussion of two key themes; carers as expert partners in care, and carers as patients.

\section{Methods}

The project team invited the views of carers via a short questionnaire survey about their experiences of primary care services. All carers known to the NHS and social care organisations in the county were invited. The questionnaire asked carers to reflect on any changes in the support that they and the person that they cared for received via GP services during the time that the CSDS site had been in operation (approximately eighteen months). The views shared via this medium offered rich insights on areas of support offered to carers through general practice, and issues where improvement could be made.

Data were also collected from GP practices in the County. These GP practices were surveyed in order to gather baseline information of the support that was offered to carers (the dissemination of this survey coincided with the timeframe that carers were asked to reflect on). A total of 57 practices were identified for the survey. The questions focussed on the services available to carers from their GP practice. 21 practices provided information (37\% response rate).

\section{Analysis}

Responses to both surveys were entered onto an electronic spread sheet and $10 \%$ independent error checking was completed. Descriptive statistics were used to summarise the data.

In addition to the closed questions posed to carers in the postal surveys, respondents were offered the opportunity to share any additional views in an open comments section ('Is there anything else that you would like to tell us about your experience, as a carer, of primary care services [GP practices]?'). These comments were subjected to the matic analysis. The initial coding was undertaken by a single member of the research team and sections of coded data were independently reviewed.

\section{Ethical review}

In the UK, studies defined as evaluations do not normally require formal NHS ethical approval. This project was defined as an evaluation by the local NHS Research and 
Development Lead and therefore the protocol was vetted by a University Research Ethics Committee. Full approval was obtained.

\section{Results}

\section{Sample}

The carer survey was sent to 1,797 carers who were recorded on carer databases held by a local third sector carer support organisation. The GP survey was distributed to 57 practices across the County and responses were drawn from practices of varying sizes, with between two and 12 GPs per practice (the mean number of GPs per practice was $6.1, \mathrm{sd}=3.2)$. Tables

\begin{tabular}{|llllll|}
\hline & & $\begin{array}{l}\text { Date of } \\
\text { distribution }\end{array}$ & Sample & $\begin{array}{l}\text { No. of } \\
\text { respondents }\end{array}$ & $\begin{array}{l}\text { Response } \\
\text { rate (\%) }\end{array}$ \\
\hline $\begin{array}{l}\text { Carer } \\
\text { survey }\end{array}$ & $\begin{array}{l}\text { Change over } \\
\text { time (18 } \\
\text { months) }\end{array}$ & August 2011 & $\begin{array}{l}1,797 \\
\text { carers }\end{array}$ & 552 & 31 \\
& & & & \\
\hline $\begin{array}{l}\text { GP } \\
\text { survey }\end{array}$ & $\begin{array}{l}\text { Audit of current } \\
\text { services }\end{array}$ & $\begin{array}{l}\text { October } \\
2011\end{array}$ & $\begin{array}{l}57 \\
\text { practices }\end{array}$ & 21 & 37 \\
\hline
\end{tabular}

Table 1 summarises the response rate of the surveys.

\section{[TABLE 1]}

Carers responding to the questionnaire survey associated themselves with a range of caring responsibilities, illustrating the diversity of their experiences. Some cared on a full-time live-in basis whilst others supported the health needs of a relative living in assisted living or residential care. Further, carers recording their experiences noted a range of relationships with the people that they cared for. Carers held caring roles for parents, a spouse, a son or daughter (both children and adults). In addition a small number of carers noted that they were in a dual-caring relationship (e.g. where a husband and wife carried out caring responsibilities for each other).

What follows is an account of the findings drawn from the carer and GP surveys which relate to General Practice support offered to carers, both in relation to their c a ring role, and their own needs as individuals/ patients. The carer survey asked carers to comment on whether the services that they received had worsened, stayed the same or improved during the preceding 18 months (the timeframe that the initiative had been in operation).

\section{Carer identification}


A number of questions in both the GP and carers surveys focussed on aspects of support from general practice that carers received in their role as carers. Examples from the carer survey included whether their surgery was aware of their caring status, if carers felt that their caring role was taken into account in their dealings with general practice, and the extent to which they were involved in the care of the person that they cared for.

Carers were asked whether their GP surgery was aware of their caring status. Alt hough $43 \%$ of carers reported that their practice had always known that they were a carer, a further $25 \%$ reported that their practice had become aware in the last 18 months. These figures suggest a considerable improvement during the previous 18 month period. Eighty-six per cent of GP practices surveyed during the study reported that they had a protocol in place for identifying carers; over $50 \%$ reported that patients' carers were identified on the patient's record and $43 \%$ reported that patients' caring responsibil it ies were always recorded on their records.

Carers were also asked whether they felt that their caring role was taken into account by the doctor or nurse at their GP practice. Fifty-two percent did not know whether their caring role was taken into account and $22 \%$ felt that there had been no change during the preceding 18 month period. The importance of a carer's role being recognized by professionals was also represented in reflections of their interactions with healthcare professionals. Parents who provided care for a child with a disability, for example, highlighted tensions where the additional issues related to providing care for someone with increased needs were not considered by healthcare staff during their interactions with them. Similar thoughts were also forthcoming from those who provided care for a spouse:

The fact that I care for 2 autistic children is irrelevant to everything, no help, no support, no understanding, oblivious to the fact that I am a carer, very poor. They do not see parents as carers, just parents.

I mentioned being the carer for a disabled child earlier in the year - was told 'all parents are carers'. Haven't broached the subject since as felt completely misunderstood.

There is no real evidence that my role as a carer is distinctly recognised at my GP practice (perhaps my role is taken for granted as I am the husband of the person cared for).

In addition, a key theme arising from the open-text question on the carer survey signalled the importance of practice staff understanding the needs of carers when they made contact. In particular carers highlighted the challenges experienced in making their needs known to reception staff: 
Receptionists need urgent training in how to help and deal with carers.

Receptionists do not take any notice of situation which can make the visit to the practice stressful for both carer and Alzheimer's patient.

I am not treated any differently to any other patient. When I had cause to complain it was not taken seriously. No extra consideration is given and a receptionist laughed at my lack of availability for appointments.

There are therefore potential impacts of this issue for both carers and those who are cared for.

\section{Carers as expert partners in care}

Carers were asked whether practice staff involved them in the care of the person that they cared for. Ninety per cent $(n=446)$ of respondents answered this question. Fifty eight per cent reported that the practice always had done this, compared to $33 \%$ of practices who never had. Ten per cent $(n=43)$ said that the practice had started to do this in the last 18 months.

An important aspect of involving carers related to correspondence between the patient and surgery. Carers were asked whether they were sent a copy of the letters that the practice of the person that they cared for sent when referring them to other services. Eighty-two percent of respondents $(n=411)$ responded to this question. Sixty-six per cent of those responding to this question reported that this had never happened, $30 \%$ that it had always happened and a further $4 \%(n=16)$ that it had started to happen in the last 18 months, illustrating limited change during the evaluation timeframe.

In turn, general practices were asked if they involved carers in discussion or decisions about the person that they cared for. Nearly half (48\%) reported that they mostly did. Seven practices (33\%) reported having an information sharing protocol in place for carer-related issues, and $43 \%$ of practices said that they sometimes copied carers into referral letters relating to the person that they cared for (whilst one -third of practices $[\mathrm{n}=7$ ] said that they never did this).

The open-text responses across each survey recounted the challenges posed where carer involvement was minimal. Some suggested that surgeries cited issues of confidentiality to justify their lack of involvement. Other carers recounted challenges and associated frustrations of attempting to define their involvement:

I do not always get information appointment times etc. They are sent to my mum mostly who tends to throw things away if she feels they are nothing to do with her. 
The person I care for has Alzheimer's dementia. If the surgery send letters regarding her mental health they send me the letter - but if it is anything to do with her physical health, e.g. healthy heart clinic or flu jabs they send the letters to her and she cannot deal with them properly and it is lucky if I find them.

A number of carers across each survey suggested that where they were not involved by healthcare staff they would try to ensure that they could access the information sent by surgeries by reviewing written correspondence sent to the person that they cared for.

Some carers noted the ways in which information sharing between themselves and practices could be improved, for example by being able to see the same GP each time. Continuity of care was considered important because it minimised the need for carers to restate the needs of those that they cared for at every consultation or to stress the importance of their involvement. Both of these issues were a source of stress for carers. Delays in obtaining appointments often resulted from attempting to see a particular GP regularly.

\section{Carers as patients}

Carers were asked a range of questions which focussed on the services that were provided to support their own needs, for example whether information about caring was made available to them, and the flexibility of practices to meet the needs of carers who needed to seek medical help in their own right (including offering home visits or flexible appointment times for carers and opportunities for carers to have a private consultation where they needed to bring the person that they cared for with them to the surgery).

The responses to the carer survey showed a trend towards carers having limited awareness of which aspects of support surgeries offered, for example $70 \%$ did not know if their practice offered home visits for carers, $63 \%$ did not know if flexible appoint ment times were provided and $67 \%$ did not know if their practice offered priority appointments for carers. Table 2 summarises these responses.

\section{[TABLE 2]}

Carer reflections on the ways in which their needs were (or were not) met were also apparent in the open text question across each of the surveys:

No one cares about carers. As long as you are able to do it, they lea ve you to get on with it. When you take ill, no one cares, you have to manage. 
The person I care for goes to a day centre three days per week from 10am-3pm. It is very difficult to get an appointment to see a doctor within those times...I cannot bring the person I care for with me, as he is too violent.

Additionally, some noted improvements that had been observed during the evaluation period:

It is easier to get appointments, flu jabs are offered earlier.

Carers'leaflets and information on a board have been prepared within the last 18 months.

Although carers cited many instances of very supportive care by individual GPs, there were others who did not feel that their own healthcare needs, or the impact on their well-being of being a carer, were understood or taken into account by healthcare professionals.

In turn, the GP survey asked practices about the range of services that were offered to carers. A high percentage of practices reported that carers had the opportunity to bring the person that they cared for to the surgery and have a private appointment, and that flexible appointments and home visits were offered for carers. Less than half of practices offered priority appointments for carers. These responses are summarised in Table 3.

\section{[TABLE 3]}

In addition, practices were asked to share information about any ot her carer support initiatives that were in place at their practices. None were offered, however one practice manager expressed surprise at the question, feeling that it wasn't the responsibility of the practice to offer this. Others cited financial pressures as a reason for not offering additional support.

\section{Discussion}

The findings of this study have highlighted a number of areas for discussion in relation to the support that is offered to carers by GP practices. The findings of the study show a considerable improvement in the extent to which carers' roles were identified by their surgeries over the 18 month period that the demonstrator site was in operation. Eighty six percent of GP practices surveyed reported that they had a protocol in place for identifying carers. This is encouraging given that the identification of carers by GPs has been cited in previous literature as a key barrier to the level of support that is offered, with up to $45 \%$ of GPs in previous studies feeling confident in being able to identify carers in their practice [13]. 
In this study, under half of the surveyed GP practices reported that the caring responsibilities of carers were noted on their patient records. Whilst responses to the carer survey suggest improvement in the identification of carers, there is a need to ensure that this information is recorded and used in ways which benefit carers. Where clinical staff are not aware of the caring responsibilities of patients, their access to effective services which meet their needs can be inhibited [14]. Over half of those replying to the carer survey did not know whether their caring role was taken into account by clinical staff when they visited for an appointment.

At the outset of this paper we signalled the significance of the contributions that carer make at an individual and community level. Their expertise and knowledge of those that they care for is key to ensuring personalised care. Fully supporting carers in their role is therefore fundamental to the needs of the person that they care for, who is ultimately vulnerable to the type of care that their friend or relative offers $[15 ; 16]$. A study in the Netherlands [17] found that carers of individuals with mental health issues reported a lack of information, consultation and support. These issues were perceived to hinder their active participation in the treatment of the person that they cared for.

Encouragingly, $10 \%$ of carers had noticed an improvement in the extent to which they were involved in the care of the person that they cared for during the 18 months that the demonstrator site had been in operation. Many published papers discuss the challenges associated with professional/ carer relationships and which impact upon the extent to which carers are involved in practice. The theme of 'conflic ting agendas' has been used, for example, in relation to the communication observed between informal caregivers and mental health staff in Australia [18; 19]. Carers in their study discussed limited opportunities for them to participate in planning support for the person that they supported. A key issue here was the barriers that they experienced in relation to unwillingness from some health staff - in this case nurses - to support participation; an issue which was also replicated in the current study.

Services which support carers to maintain their own health are clearly prioritised in both national and local level strategies in the UK. As we have discussed above, carers may experience a range of medical problems which relate specifically to their caring role [1]. Where supportive services which enable them to maintain their health are not forthcoming this may lead to undesirable outcomes not only for themselves, but also for the person that they care for. The importance of recognising the needs of carers as individuals - and patients - in their own right, is therefore of utmost importance. Previous research has, however, signalled the issues associated with Genera I Practices supporting carers effectively, suggesting that whilst GPs often have an awareness of the 
emotional and physical impacts that caring may have, they are constrained by time and limited resources with which to identify carers $[13 ; 20]$.

Encouragingly, a high percentage of practices responding to our survey reported that home visits, priority appointments and the opportunity to bring the person that they cared for to the surgery and still have a private consultation were offered. Conversely, large numbers of carer respondents did not know whether these services were offered by their surgery. In 2005 a detailed review of studies which considered potential barriers to carers accessing health services in their own right was completed by the National Coordinating Centre for the Service Delivery and Organisation Programme [12]. They identified five issues of particular importance in this area including professional response (professionals showing a lack of understanding towards carers needs as carers), service organisation and delivery (services did not record patients' caring responsibilities or provide for them), language or culturally held beliefs and practices (e.g. non-English speaking carers could not communicate their emotional needs), characteristics of carers (carer's approach, behaviour and values in accessing services), and lack of information and knowledge (lack of information in relation to support). It is not possible to compare the responses of carers directly to the services that were offered by their ow $n$ practice, however, a broad comparison of the services offered by GP practices, and the practices cited as available by carers suggests that there is room for improvement in the methods that practices use to share information about the services that they provide.

Carers' use of GP services will likely change at any given time according to the a mo unt of care that they provide, who they are providing it for, and the length of time that they have been undertaking these duties [14]. This, coupled with an awareness that information provided to carers by health and social care organisations often varies in quality and accessibility [21]. Accessible information which is regularly updated is therefore of paramount importance in supporting carers to support themselves, and those that they care for [22].

\section{Conclusion}

Whilst this study was completed as part of a wider evaluation of the national roll out of the Carer Demonstrator Sites in the UK, it has signalled a range of areas for improvement in supporting carers with their needs. The detailed information gleaned from a large number of carers, and a range of GP practices, have highlighted the importance of practices identifying where their patients have caring responsibilities - and where their patients have an informal/ familial carer. The evaluation of the demonstrator site pilot began to show important improvements in this area, however work is still 
needed to ensure that, following collation of this information, a targeted response to carer support is implemented. In addition effective methods of publicising available services to carers are essential to ensure that they are supported both as caring experts, and as individuals with diverse and changing needs. 


\section{Acknowledgements}

This study was funded by NHS Northamptonshire. We are grateful to the commissioners, to Northamptonshire Carers who supported its completion, and to the hundreds of carers who so passionately shared their views. 


\section{References}

1. HM Government (2008) Carers at the heart of 21st-century families and communities. The United Kingdom Carers Strategy. London: Department of Health.

2. CIRCLE (2011) New Approaches to Supporting Carers' Health and Well-being: Evidence from the National Carers' Strategy Demonstrator Sites programme (Final report). Available at: http://www.sociology.leeds.ac.uk/assets/files/Circle/newapproaches-report-jan-12.pdf Accessed: 18-09-12.

3. Fox, A. (2011) The Health Case for supporting carers. The Princess Royal Trust for Carers. Available from: http://static.carers.org/files/health-case-for-carer-supportjan09b-3928. doc. Accessed: 01-02-11.

4. Dearden, C. and Becker, S. (2004) Young Carers in the UK: The 2004 report. London: Carers UK.

5. Lawrence, V., Murray, J., Samsi, K. and Banerjee, S. (2008) Attitudes and support needs of black Caribbean, south Asian and white British carers of people with dementia in the UK. The British Journal of Psychiatry, 93, 240-246.

6. Niblett, P. (2010) Personal Social Services Survey of Adult Carers in England - 200910. The NHS Information Centre, Social Care.

7. Prior, L. (2003) Belief, knowledge and expertise: the emergence of the lay expert in medical sociology. Sociology of Health and Illness, 25, 41-57.

8. Shaw, J. and Baker, M. (2004) "Expert patient" - dream or nightmare? BMJ, 328, 723-724.

9. Department of Health (2010) The Handbook to the NHS Constitution. London: Department of Health.

10. Elwick, H. Joseph, S., Becker, S. and Becker, F. (2010) Manual for the Adult Carer Quality of Life Questionnaire (AC-QoL). The Princess Royal Trust for Carers/ The University of Nottingham.

11. Buckner, L. and Yeandle, S. (2005) Older carers in the UK. London: Carers UK.

12. National Coordinating Centre for the Service Delivery and Orga nisation (NCCSDO) research programme (2005) Briefing paper: Access to health care for carers. London: NCCSDO.

13. Greenwood, N., Mackenzie, A., Habibi, R., Atkins, C. and Jones, R. (2010) General practitioners and carers: a questionnaire survey of attitudes, awareness of issues, barriers and enablers to provision of services. BMC Family Practice, 11, 100.

14. Arksey, H., and Hirst, M. (2005) Unpaid carers' access to and use of primary care services. Primary Health Care Research and Development, 6(2), 101-116.

15. Oyebode, J. (2003) Assessment of carers' psychological needs. Advances in Psychiatric Treatment, 9, 45-53.

16. Kalra, L., Evans, A., Perez, I., Melbourn, A., Patel, A., Knapp, M. and Donaldson, N. (2004) Training carers of stroke patients: randomised controlled trial. BMJ, 328, 1-5. 
17. van de Bovenkamp, H. M. and Trappenburg, M. J. (2010) The relationship bet ween mental health workers and family members. Patient Education and Counselling, 80, 120125.

18. Goodwin, A. and Happell, B. (2006) Conflicting agendas between consumers and carers: The perspectives of carers and nurses. International Journal of Mental Health Nursing, 15, 135-143.

19. Goodwin, A. and Happell, B. (2007) Consumer and carer participation in mental health care: The carer's perspective: Part 2 - Barriers to effective and genuine participation. Issues in Mental Health Nursing, 28, 625-638.

20. Jones, R., Mackenzie, A., Greenwood, N., Atkins, C., and Habibi, R. (2011) General practitioners, primary care and support for carers in England: can training make a difference? Health and Social Care in the Community, 20(2), 128-136.

21. Manthorpe, J., Moriarty, J., Cornes, M., Hussein, S. and Lombard, D. (2013). On-line information and registration with services: patterns of support for carers in England. Working with Older People, 17(3), 125-129.

22. Jarvis, A. and Worth, A. (2005) Meeting carers' information needs. Community Practitioner, 78(9), 322-326. 
Tables

\begin{tabular}{|llllll|}
\hline & & $\begin{array}{l}\text { Date of } \\
\text { distribution }\end{array}$ & Sample & $\begin{array}{l}\text { No. of } \\
\text { respondents }\end{array}$ & $\begin{array}{l}\text { Response } \\
\text { rate (\%) }\end{array}$ \\
\hline $\begin{array}{l}\text { Carer } \\
\text { survey }\end{array}$ & $\begin{array}{l}\text { Change over } \\
\text { time (18 } \\
\text { months) }\end{array}$ & August 2011 & $\begin{array}{l}1,797 \\
\text { carers }\end{array}$ & 552 & 31 \\
& & & & \\
\hline $\begin{array}{l}\text { GP } \\
\text { survey }\end{array}$ & $\begin{array}{l}\text { Audit of current } \\
\text { services }\end{array}$ & $\begin{array}{l}\text { October } \\
2011\end{array}$ & $\begin{array}{l}57 \\
\text { practices }\end{array}$ & 21 & 37 \\
\hline
\end{tabular}

Table 1: survey responses 


\begin{tabular}{|c|c|c|c|c|}
\hline Question & $\begin{array}{l}\text { Yes, } \\
\text { always } \\
\text { has } \\
\%(n)\end{array}$ & $\begin{array}{l}\text { Yes, } \\
\text { provided } \\
\text { in last } 18 \\
\text { months \% } \\
\text { (n) }\end{array}$ & $\begin{array}{l}\text { No, } \\
\text { never } \\
\text { has } \\
\% \text { (n) }\end{array}$ & $\begin{array}{l}\text { Don't } \\
\text { know } \\
\%(n)\end{array}$ \\
\hline $\begin{array}{l}\text { Does your Practice offer home } \\
\text { visits for carers? }\end{array}$ & $7(34)$ & $1(6)$ & $22(113)$ & $70(353)$ \\
\hline $\begin{array}{l}\text { Does your Practice offer flexible } \\
\text { appointment times for carers? }\end{array}$ & $10(51)$ & $3(17)$ & $24(120)$ & $63(320)$ \\
\hline $\begin{array}{l}\text { Does the practice offer priority } \\
\text { appointments for carers? }\end{array}$ & $3(13)$ & $3(13)$ & $27(140)$ & $67(343)$ \\
\hline $\begin{array}{l}\text { Can you bring the person you } \\
\text { care for to the surgery and still } \\
\text { have a private consultation? }\end{array}$ & $33(162)$ & $1(6)$ & $14(67)$ & $52(251)$ \\
\hline
\end{tabular}

Table 2: Carers as patients: summary of responses 


\begin{tabular}{|lll|}
\hline Service & Frequency & \% of practices \\
\hline Home visits for carers & 14 & 67 \\
\hline Flexible appointment times for carers & 16 & 76 \\
\hline Priority appointments for carers & 10 & 48 \\
\hline $\begin{array}{l}\text { Bringing cared for person to surgery and still } \\
\text { having a private consultation }\end{array}$ & 18 & 86 \\
\hline
\end{tabular}

Table 3: Services offered by GP practices to carers 